\title{
Mastectomía de Rescate, prevalencia y alternativas de Reconstrucción Mamaria.
}

\section{Rescule Mastectomy, prevalence and Breast Reconstruction alternatives.}

\section{Pablo Ulloa Ochoa1, Antonio Jurado Bambino*2, Edgar Pilco A, Luis Morejón Naranjo², Gladis Mora Veintimilla4.}

1. Universidad de Especialidades Espíritu Santo (UEES). Samborondón, Guayaquil-Ecuador.

*Correspondencia:

antonio_jurado@yahoo.com

Teléfono [593] 0987678335

Conflicto de intereses: Los autores declaran no tener conflictos de intereses.

Fondos: Ver la página 207

Recibido: 13 Diciembre 2016 Aceptado: 20 Mayo 2017

Publicado: 30 Diciembre 2017

Membrete bibliográfico: Ulloa $P$, Jurado A, Pilco E, Morejón L, Mora G. Rev. Oncol. Ecu 2017;27(3):200-211

DOI: https://doi.org/10.33821/226

Copyright Ulloa, et al. Este artículo es distribuido bajo los términos de Creative Commons Attribution License, el cual permite el uso y redistribución citando la fuente y al autor original.
2. Servicio de Mastología, Instituto Oncológico Nacional “Dr. Juan Tanca Marengo” Solca-Guayaquil.

3. Área de Emergencia, Hospital General Guasmo Sur. Guayaquil.

4. Carrera de Enfermería, Universidad Técnica de Machala.

\section{Resumen}

Introducción: La Mastectomía de rescate (MR) es la técnica quirúrgica que está indicada en pacientes con recidiva local posterior a tratamiento conservador y con poca respuesta a la neoadyuvancia. El objetivo de este estudio es presentar una serie de casos de Mastectomía de rescate, describir los colgajos utilizados en estas cirugías y la prevalencia de este tipo de cirugía.

Métodos: El presente estudio descriptivo y retrospectivo, recolecta información de pacientes atendidos en el Servicio de Mastología de Solca- Guayaquil durante el período de enero 1 al 31 de diciembre del 2015. Las cuales reportaban diagnóstico histopatológico de carcinoma mamario y cursaban clínicamente en estadio IIb, III y IV. Se utiliza estadística univariada.

Resultados: La prevalencia de la MR fue de 14/610 casos (5.32\%). En la presente serie de casos el 57 \% fueron resueltos con colgajos a tensión y el 14 \% fue resuelto con colgajo dorsal ancho. El resultado final a los 18 meses post cirugía 3 pacientes fallecieron, 3 pacientes sin actividad tumoral, y 6 pacientes con pérdida de seguimiento. La mayor incidencia de casos según su histología fue el carcinoma ductal infiltrante.

Conclusión: La analgesia intratecal con morfina en 15 pacientes con cáncer terminal, mejora la calidad de vida de $27.6 \%$ a $88.2 \%$ en un lapso de 3 meses y la calidad de vida se mantiene hasta 90 días.

Palabras Claves: NEOPLASIAS DE LA MAMA, MASTECTOMÍA, MAMOPLASTIA. 


\section{Abstract}

Introduction: Rescue Mastectomy (RM) is the surgical technique that is indicated in patients with local recurrence after conservative treatment and with little response to neoadjuvant therapy. The aim of this study is to present a series of rescue mastectomy cases, describe the flaps used in these surgeries and the prevalence of this type of surgery.

Methods: This descriptive and retrospective study collects information from patients treated at the Mastology Service of Solca-Guayaquil during the period from January 1 to December 31, 2015. They reported histopathological diagnosis of mammary carcinoma and were enrolled clinically in stage IIb , III and IV. Univariate statistics are used.

Results: The prevalence of MR was $14 / 610$ cases (5.32 \%). In the present case series, $57 \%$ were resolved with tension flaps and $14 \%$ was resolved with a latissimus dorsi flap. The final result at 18 months after surgery 3 patients died, 3 patients without tumor activity, and 6 patients with loss of follow-up. The highest incidence of cases according to their histology was infiltrating ductal carcinoma.

Conclusion: RM has a low prevalence, in this series most cases were resolved with tension flaps.

Keywords: BREAST NEOPLASMS, MASTECTOMY, MAMOPLASTY.

DOI: $10.33821 / 226$

\section{Introducción}

La mastectomía de rescate (MR) es la técnica quirúrgica realizada con intenciones curativas en un paciente portador de cáncer de mama recidivante luego de una primera intervención con Mastectomía conservadora y la utilización de quimioterapia y radioterapia $[1,2]$. Debido a que esta segunda cirugía se realiza en un episodio de recurrencia, la cirugía de MR se constituye un desafío por la alteración anatómica dada por el tumor y por la cirugía previa. El manejo de estos pacientes debe ser multidisciplinario ya que el tumor generalmente sobrepasa los $5 \mathrm{~cm}$ y, habitualmente el paciente es sometido a quimioterapia neoadyuvante con antraciclinas y taxanos con o sin traztuzumab según estado de HER2, lo que podría tener consecuencias con el proceso de cicatrización del tejido, considerando que el objetivo incluye remover el tumor recidivante con márgenes negativos [1, 2].

El cierre y la reconstrucción primaria debido a la radicalidad de esta cirugía, está condicionada por varios factores como los tratamientos previos, el estado de la piel, los músculos y el tejido subcutáneo del área operatoria. Sin embargo existen varias alternativas y técnicas quirúrgicas que pueden ser usadas como el cierre primario, el injerto libre de piel, colgajos locales y la rotación de colgajos. Dentro de estos últimos se han descrito mamarios, de avance bilateral, toracoepigástrico, toracoabdominal y los colgajos miocutáneos. Los colgajos miocutáneos tienen una gran aplicación ya que adicionalmente da una solución estructural por el volumen de reemplazo dado por el músculo. Los colgajos miocutánoeos más utilizados se han descrito como recto anterior del abdomen (TRAM) [3], Dorsal Ancho (Lat Dorsi), Pectoral Mayor y el oblicuo externo (DIEP) el cual proviene de la 
parte inferior del abdomen pero solo tiene piel y grasa sin sacrificar el músculo recto abdominal $[3,4]$.

El objetivo de este estudio es presentar una serie de casos de Mastectomía de rescate, describir los colgajos utilizados en estas cirugías y la prevalencia de este tipo de cirugía.

\section{Materiales y Métodos}

En el área de Mastología del Instituto Oncológico Nacional (ION), durante el periodo de 1 de Enero del 2015 al 31 Diciembre del 2015, se realizó un estudio descriptivo, retrospectivo, considerando a 610 pacientes con diagnóstico clínico y preoperatorio de cáncer de mama, la población fue en base a los criterios de inclusión y exclusión.

Criterios de inclusión:

1. Pacientes sometidas a mastectomía de rescate o limpieza en el año 2015.

2. Pacientes que recibieron la neoadyuvancia y adyuvancia en ION Guayaquil.

3. Pacientes intervenidas quirúrgicamente en el ION Guayaquil.

Criterios exclusión

1. Datos incompletos en sistema.

2. Menores de 20 años y mayores de 80 años

Se estudiaron las variables: edad, región anatómica de ubicación del tumor inicialmente, estadio clínico en la primera consulta, clasificación histopatológica, protocolo de la neoadyuvancia, respuesta a la neoadyuvancia y tipo de cierre. El análisis estadístico fue medidas de tendencia central los resultados fueron presentados con diagramas de barras.

\section{Resultados}

\section{Prevalencia de Mastectomía de rescate}

De un total de 610 casos de cáncer de mama diagnosticados y tratados en el ION 2015 se sometieron a mastectomía 263 pacientes de los cuales 14 casos (5.32\%) fueron de rescate, siendo $2.30 \%$ del total de casos diagnosticados. Se presentan los datos descriptivos de la población en la tabla 1.

\section{Casos de mastectomía de rescate}

El grupo etario más frecuente fue comprendido entre los 40 a 65 años (9 pacientes), con volumen tumoral mayor de $5 \mathrm{~cm}$ en todos los casos. El $36 \%$ de los pacientes el tumor ocupaba casi todo el seno, seguido de un $21 \%$ el cuadrante supero externo. A la primera consulta fueron calificadas un 57 \% con estadio IV. El diagnóstico histopatológico reveló el 
carcinoma ductal infiltrante fue el más frecuente con un $86 \%$. Los receptores estrogénicos fueron positivos en 8 de los 14 pacientes, y los receptores de progesterona tuvieron igual porcentaje para los positivos y negativos, HER2NEU fue positivo en 8 de los pacientes, y el Ki67 fue positivo con más de $50 \%$ en 7 de los 14 casos. El $79 \%$ de los pacientes fueron sometidos a neoadyuvancia. El protocolo más utilizado fue paclitaxel 12 ciclos + FAC 4 ciclos con un 33 \% (Figura 1). En la evaluación previa a la cirugía se evidencia la respuesta a la quimioterapia, un $42 \%$ de los pacientes con una respuesta mayor al $50 \%$ en relación al tamaño tumoral, el $17 \%$ de los pacientes demostraron una respuesta menor al $50 \%$, el $25 \%$ de los pacientes reportaron progresión de la enfermedad durante la neoadyuvancia y ningún paciente tuvo una respuesta total a la neoadyuvancia.

El uso de Gencitabina en uno de los casos, se documentó la mayor toxicidad produciendo falla renal. Los demás pacientes que siguieron los esquemas actuales reportaron una respuesta aceptable a la quimioterapia con menor toxicidad. Los pacientes que usaron el protocolo con Adriamicina y ciclofosfamida no interfirieron en el tratamiento y tuvieron una buena respuesta.

\section{Cirugía de Mastectomía de rescate}

Todos los pacientes fueron sometidos a mastectomía de rescate, el $57 \%$ de los casos su cierre fue a tensión sin utilización de colgajos, el 23 \% de los pacientes su cierre se utilizó un colgajo de avance que fue toracoepigástrico, colgajo miocutáneo utilizado fue el dorsal ancho con un $14 \%$ (Figura 2).

En lo que respecta a la adyuvancia un $79 \%$ fueron sometidas a quimioterapia y un $64 \%$ a radioterapia. En relación a la situación actual de los pacientes un $43 \%$ se las califica como perdidas de las consulta en relación a la fecha de la última cita reportada, $21 \%$ fallecidas, $7 \%$ viva con recidiva, $7 \%$ viva con metástasis, $21 \%$ libres sin evidencia de metástasis o recidiva. 
Tabla 1. Descripción de la serie de casos

\begin{tabular}{|c|c|c|}
\hline Variable & & Casos \\
\hline \multicolumn{3}{|l|}{ Edad } \\
\hline & $<40$ años & 1 \\
\hline & 40 a 65 años & 9 \\
\hline & $>65$ años & 4 \\
\hline \multicolumn{3}{|l|}{ Estadio } \\
\hline & II & 4 \\
\hline & III & 2 \\
\hline & IV & 8 \\
\hline \multicolumn{3}{|c|}{ Tipo Histológico } \\
\hline & Ductal infiltrante & 12 \\
\hline & Lobulillar & 1 \\
\hline & Otro & 1 \\
\hline \multicolumn{3}{|c|}{ Región anatómica } \\
\hline & CSE & 3 \\
\hline & CSI & 1 \\
\hline & $\mathrm{CIE}$ & 1 \\
\hline & CII & 2 \\
\hline & Retroareolar & 2 \\
\hline & Tota la mama & 5 \\
\hline \multicolumn{3}{|c|}{ Neoadyuvancia } \\
\hline & Si & 12 \\
\hline & No & 2 \\
\hline \multicolumn{3}{|c|}{ Respuesta a la Neoadyuvancia } \\
\hline & $100 \%$ & 0 \\
\hline & $>50 \%$ & 5 \\
\hline & $<50 \%$ & 2 \\
\hline & Estable & 2 \\
\hline & Progresión & 3 \\
\hline \multicolumn{3}{|c|}{ Situación actual de la paciente a los 18 meses postquirúrgico. } \\
\hline & Sin actividad tumoral & 3 \\
\hline & Recidiva & 1 \\
\hline & Metástasis & 1 \\
\hline & Fallece & 3 \\
\hline & Pérdida de seguimiento & 6 \\
\hline
\end{tabular}


Figura 1. Protocolos de quimioterapia utilizados

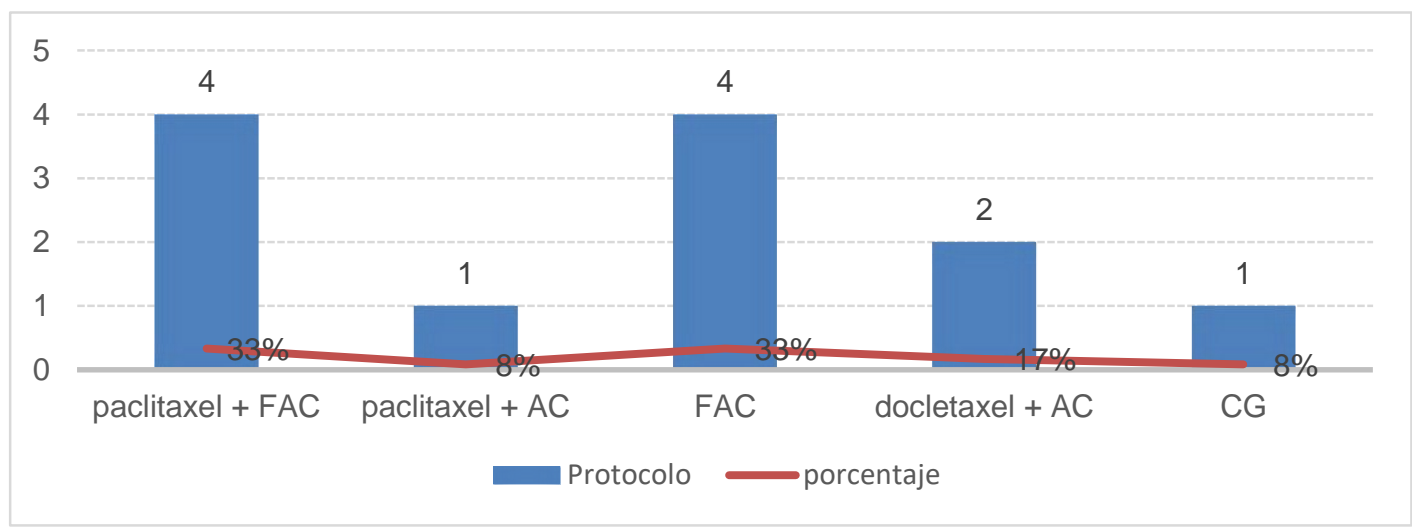

Figura 2. Tipos de cierre posterior a mastectomía

\section{Tipo de cierre}
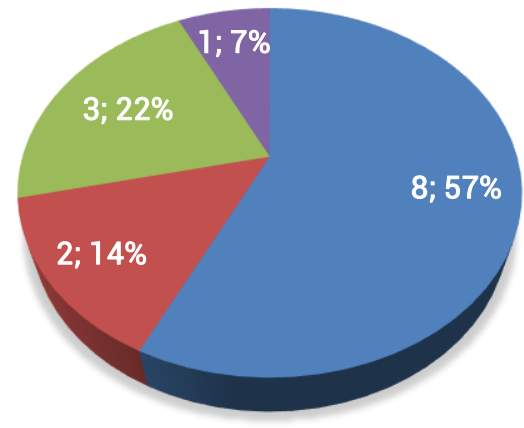

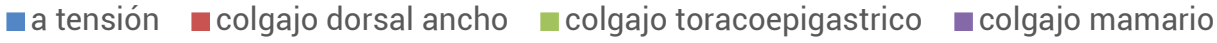

\section{Discusión}

En el presente estudio se describe que la prevalencia de MR es del $5.3 \%$. La MR se realizó en 14 casos, el $57 \%$ fueron resueltos con colgajos a tensión y el $14 \%$ fue resuelto con colgajo dorsal ancho. El resultado final a los 18 meses post cirugía 3 pacientes fallecieron, 3 pacientes sin actividad tumoral, y 6 pacientes con pérdida de seguimiento.

Es importante determinar el estadio clínico del carcinoma mamario en la primera consulta. El diagnóstico ante la sospecha clínica de la enfermedad que puede apoyarse en exámenes de laboratorio e imagenológicos. Dentro del proceso terapéutico, dos pacientes no aceptaron neoadyuvancia, uno de ellas reportó recidiva y otro paciente con pérdida de seguimiento, mientras que los que se sometieron a neoadyuvancia tuvieron una respuesta variada ante los esquemas utilizados; en un trabajo descrito previamente se indica como 
factor de buen pronóstico el tratamiento con quimioterapia de neoadyuvancia en 220 pacientes a diferencia de las que no lo aceptaron fueron 36 casos resultados comparables con esta serie de casos [5]. El uso de Gencitabina en uno de los casos, se documentó la mayor toxicidad produciendo falla renal, esto representa un atraso el tratamiento quirúrgico, lo cual está en relación a un estudio en la que se reportó mayor toxicidad a nivel pulmonar posterior al uso de este fármaco [6]. Los demás pacientes que siguieron los esquemas actuales reportaron una respuesta aceptable a la quimioterapia con menor toxicidad, como lo describe Portelles et al, que el protocolo con Adriamicina y ciclofosfamida no interfirió en el tratamiento y tuvieron una buena respuesta [7]. En lo que respecta a una visión global de las pacientes fallecidas en la inmunohistoquímica reportaban Ki67 positividad mayor del $50 \%$, Her2NEU positivo en dos de los tres pacientes, comparado con el estudio de Arrechea et al, de 272 pacientes 27 presentaban Her2NEU+ evidenciándose en ellos el peor pronóstico [8].

En lo que respecta a la linfadenectomía 2 de las 3 pacientes sin recidiva no presentaron ganglios comprometidos, Gonzales et al, en su revisión de 273 pacientes reporta que la invasión vascular y linfática es inversamente proporcional a la supervivencia [9].

La presente serie reportó que dos de los pacientes libres tenían receptores hormonales positivos, como se indica en el estudio de Gonzales et al, que los receptores de estrógenos y progesterona positivos se convierten en factores de buen pronóstico [9].

Con respecto al cierre a pesar de la gran tensión de los bordes de los 8 pacientes ninguno presentó necrosis de los bordes permanente, o necesidad de ser reintervenida. De los pacientes que se sometieron al uso de colgajos, todos fueron aceptados y hubo un buen cierre estético y un considerable grado de satisfacción, semejante al estudio de Vásquez et al, en el que describe la aplicación de distintitas técnicas oncoplásticas generando satisfacción, disminuyendo la morbilidad, sin ser un impedimento para el inicio de al adyuvancia [10].

\section{Conclusiones}

La MR tiene una baja prevalencia menor al $6 \%$. En la presente serie de casos el $57 \%$ fueron resueltos con colgajos a tensión y el $14 \%$ fue resuelto con colgajo dorsal ancho. El resultado final a los 18 meses post cirugía 3 pacientes fallecieron, 3 pacientes sin actividad tumoral, y 6 pacientes con pérdida de seguimiento. La mayor incidencia de casos según su histología fue el carcinoma ductal infiltrante.

\section{Agradecimientos}

Se reconoce a las personas que participaron indirectamente en el estudio tales como los pacientes, como personal técnico, otras en general de ION "Dr. Juan Tanca Marengo" SOLCA-Guayaquil. 


\section{Información adicional}

\section{Abreviaturas}

CSE: Cuadrante supero externo.

CSI: Cuadrante supero interno.

Nota del Editor

CIE: Cuadrante inferior externo

La Revista Oncología Ecu

CII: Cuadrante inferior interno.

permanece neutral con

respecto a los reclamos

MR: Mastectomía de rescate.

Solca: Sociedad de Lucha Contra el Cáncer.

jurisdiccionales en mapas

TRAM: colgajo miocutáneo del recto anterior del abdomen.

DIEP: colgajo del oblicuo externo.

publicados y afiliaciones

Her2NEU: receptor 2 de factor de crecimiento epidérmico humano.

\section{Archivos Adicionales}

Se presentan casos gráficos de las cirugías efectuadas.

Fondos

Los fondos para la presente investigación fueron propios de los autores del presente artículo.

\section{Disponibilidad de datos y materiales}

Existe la disponibilidad de datos bajo solicitud al autor de correspondencia. No se reportan otros materiales.

\section{Contribuciones de los autores}

PUO, AJB idea de investigación, EPA, LMN y GMV realizaron la revisión bibliográfica. PUO, EPA, LMN compilaron los datos. AJB y PUO escribieron el artículo. AJB y EPA realizaron el análisis crítico del estudio. Todos los autores aprobaron la versión final del manuscrito.

Aprobación de ética y consentimiento para participar

No aplica ya que es un estudio de revisión de historias clínicas. 


\section{Consentimiento para publicación}

No aplica.

\section{Información de los autores}

Pablo Ulloa Ochoa, Médico de Cirugía General. Universidad de Especialidades Espíritu Santo (UEES). Samborondón, Guayaquil-Ecuador.

Antonio Jurado Bambino, Cirujano Oncólogo, Médico Tratante del Servicio de Mastología del Instituto Oncológico Nacional "Dr. Juan Tanca Marengo", Solca-Ecuador. Telef.: 0987678335 -E-mail: antonio_jurado@yahoo.com

Edgar Pilco A, Cirujano General. Subjefe de Guardia. Área de Emergencia Hospital General Guasmo Sur. Guayaquil. Telef.: - 0982039104. E-mail: enrique_pilco@hotmail.com

\section{Luis Morejón Naranjo, Cirujano General.}

Gladis Mora Veintimilla, Licenciada en enfermería. Docente titular de carrera de Enfermería - Universidad Técnica de Machala.

\section{Revisiones por pares}

Acceda a la revisión de pares académicos en el siguiente enlace: https://publons.com/review/3162370

\section{Referencias}

Abreviaturas en la referencias DOI: Digital Object Identifier

PMID: PubMed Identifier SU: Short URL
1. Hernández G, Arcia F, Acosta V, Troconis J, Ferri N, Betancourt L, et al. Cáncer de mama estadios I y II, Reunión de Consenso. Rev Venez Oncol. 2006;18(2):125-133.

2. Iglesis $R$, Cabello $R$, Fontbona $M$, Baeza $R$, Amar $M$. Cirugía oncoplástica para cáncer mamario: mejorando estéticamente la resecabilidad. Rev. chil. obstet. ginecol. 2011; 76(1): 32-36. SU: goo.gl/9yiMex

3. Ospino R, Cendales R, Cifuentes J, Sánchez Z, Galvis J, Bobadilla I. Supervivencia en pacientes con cáncer de mama localmente avanzado tratadas con radioterapia posterior a mastectomía en el Instituto Nacional de Cancerología. Rev Colomb Cancerol. 2010; 14(4): 210-224.

4. Mohar A, Bargalló E, Ramírez MT, Lara F, Beltrán-Ortega A. Recursos disponibles para el tratamiento del cáncer de mama en México. Salud pública Méx. 2009;51 ( Suppl 2): s263-s269. SU: goo.gl/WWkso2

5. Vázquez T, Krygier G, Barrios E, Cataldi S, Vázquez, Alonso R, et al. Análisis de sobrevida de una población con cáncer de mama y su relación con factores pronósticos: estudio de 1.311 pacientes seguidas durante 230 meses. Rev. Méd. Urug. 2005;21( 2 ):107-121. 
6. Jové J, Arellano A. Toxicidad y tratamientos de soporte en oncología radioterápica: tórax y sistema nervioso central. Riesgo/beneficio de las terapias asociadas. Oncología (Barc.) 2005;28(2):71-76. SU: goo.gl/FXjvhR

7. Portelles A, Rodríguez Y, Fernández $\mathrm{P}$, Sanz N, Oller J. Quimioterapia neoadyuvante en cáncer de mama, localmente avanzado. CCM 2013;17(4):333-442. SU: goo.gl/5GscbQ

8. Arrechea M, Vicente F, Córdoba A, Ibáñez B, Santamaría M, Guillén Grima F.. Molecular subtypes of breast cancer: prognostic implications and clinical and immunohistochemical characteristics. Anales Sis San Navarra 2011;34(2):219-233. SU: goo.gl/TF9weS

9. González-Longoria L. Factores pronósticos anatomopatológicos de supervivencia en el cáncer de mama. MEDISAN 2012;16(12):1899-1905. SU: goo.gl/1vyhH3.

10. Vázquez C. Breast cancer surgery: treatment surgical techniques and reconstruction, recovery time and time. Med. segur. trab. 2016;62(Suppl):116-124. 
caso 1: Excéresis completa de glándula mamaria izquierda.
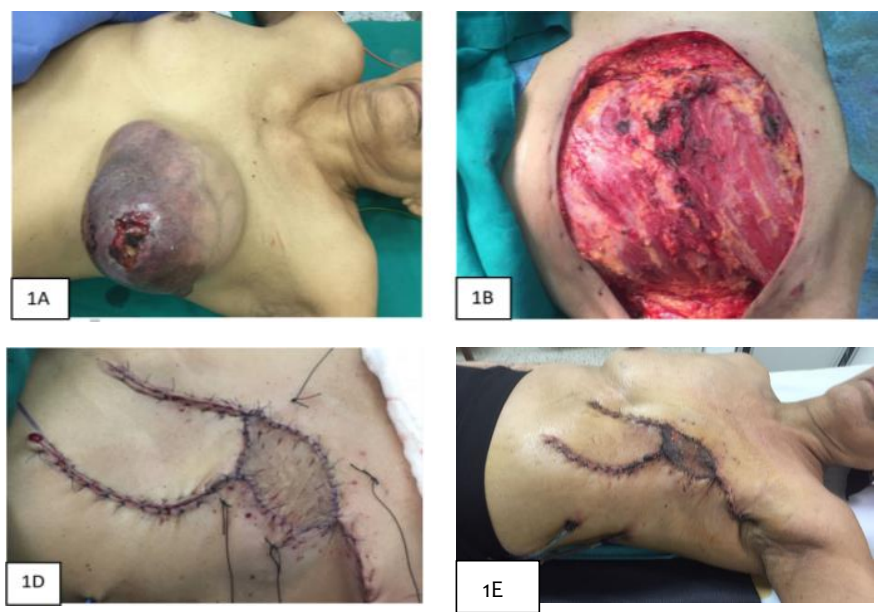
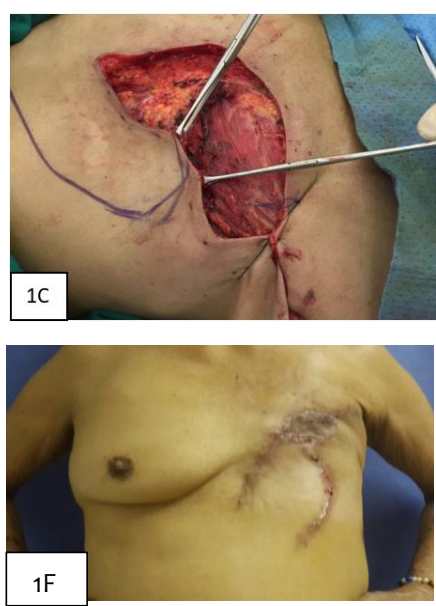

Figura 1A: Tumor de mama con pobre respuesta a la quimioterapia.

Figura 1B: Lecho quirúrgico post mastectomía ampliada de rescate con segmento muscular.

Figura 1C: Diseño de colgajo musculo cutáneo de rotación y avance.

Figura 1D: Cierre del defecto con el colgajo e injerto libre total de piel de región inguinal izquierda.

Figura 1E: Control postoperatorio a las 2 semanas.

Figura 1F: Control postoperatorio a los 3 meses sin limitación funcional

Caso 2: Carcinoma de mama derecha.
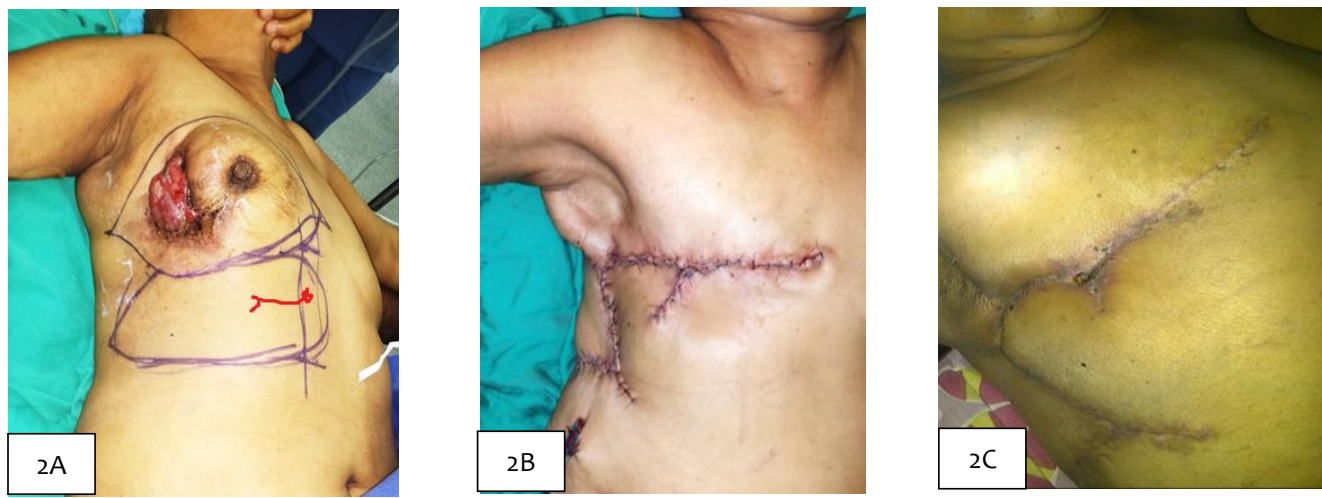

Figura 2A: Carcinoma de mama derecha respuesta parcial a la quimioterapia. Diseño del colgajo Toracoepigástrico de rotación.

Figura 2B: Postoperatorio inmediato post mastectomía de rescate.

Figura 2C: Control postoperatorio a los 3 meses. Paciente puede pasar a radioterapia 
caso 3: Tumor Exofítico de mama derecha
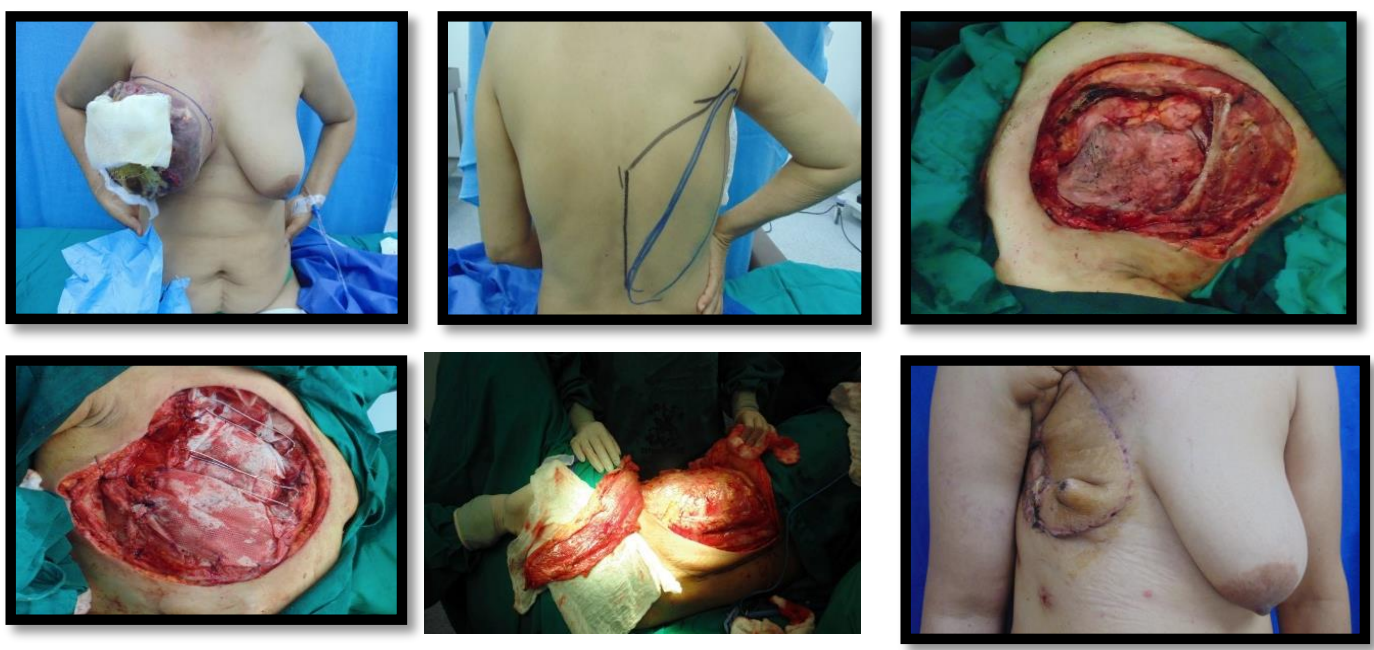

De izquierda a derecha en filas:

Figura 3A: Tumor exofítico de mama derecha recidivante inflamatorio.

Figura 3B: Diseño del colgajo de gran dorsal para reconstrucción mamaria.

Figura 3C: Infiltración del músculo pectoral mayor y de los músculos intercostales II, III, IV, V y VI, por lo que en un segundo tiempo se realiza resección parcial de la pared torácica en bloque con costillas.

Figura 3D: Toracectomía con resección de pared torácica y reconstrucción con alambre, malla, y colgajo del músculo gran dorsal.

Figura 3E:. Colgajo de gran dorsal derecho.

Figura 3F: Control posterior a 3 meses. 